\title{
Sistema electrónico para control de actuadores mediante Bluetooth
}

\section{Electronic system for control of actuators using Bluetooth}

\section{Hans Raúl Márquez Ramos}

Ingeniero Electrónico. Analista de Calidad de Q-Vision Technologies S.A. Bogotá, Colombia.

hmarquez@qvision.com.co

\section{Cesar Hernández}

Ingeniero Electrónico, magíster en Ciencias de la Información y las Comunicaciones. Docente e investigador de la Universidad Distrital Francisco José de Caldas. Bogotá, Colombia.

cahernandezs@udistrital.edu.co

\section{Luis F. Pedraza}

Ingeniero Electrónico, magíster en Ciencias de la Información y las Comunicaciones. Docente e investigador de la Universidad Distrital Francisco José de Caldas. Bogotá, Colombia.

1fpedrazam@udistrital.edu.co

Clasificación del artículo: Investigación (Recreaciones)

Fecha de recepción: 26 de abril de 2011

Fecha de aceptación: 28 de noviembre de 2011

Palabras clave: Actuadores, bluetooth, esclavo, inalámbrica, maestro.

Keywords: Actuators, bluetooth, slave, wireless, master.

\section{RESUMEN}

En este artículo se presenta el proceso de diseño de un sistema electrónico el cual controla un máximo de ocho actuadores de forma inalámbrica a través de dispositivos Bluetooth, los cuales están conformados por un módulo maestro y un módulo esclavo, siendo este último el que alberga las salidas para el control de actuadores, además de tener disponible lectura de la variable temperatura.
Se utilizó un sistema de control compuesto por un microcontrolador Motorola GP32, el cual permite escoger dos modos de operación en el que uno de ellos permite al usuario trabajar desde el computador controlando los actuadores y el otro modo de operación prescinde del computador para ejecutar el control sobre estos.

\section{ABSTRACT}

This paper presents the process of designing an 


\section{re-creaciones}

electronic system which controls up to 8 actuators wirelessly through Bluetooth devices which are composed by a master module and slave module, the latter being that contains the outputs for control of actuators available in addition to reading the temperature variable.
They use a control system comprising a GP32 Motorola microcontroller which allows you to choose two modes of operation in which one allows the user to work from the computer controlling the actuators and the other mode dispenses with the computer to execute control over these.

\section{INTRODUCCIÓN}

En los entornos industrializados el control que se ejerce sobre los diversos actuadores que se encuentran en este ambiente a menudo son alambrados, debido a que estos generalmente están asociados a controladores lógicos programables o PLC's lo cual impone una restricción en cuanto al área de maniobra por parte del operador que esta controlando un proceso mediante una máquina.

La limitante de distancia restringe al operador a ubicarse en un área cercana al proceso objetivo para supervisar el correcto funcionamiento de este. Sin embargo, el posible riesgo que conlleva la tarea de vigilancia puede tener inconvenientes para el operador ya que su integridad física está en juego.

Debido a este panorama cotidiano en las grandes industrias, este artículo describe el proyecto: "Sistema electrónico para control de actuadores mediante Bluetooth" desarrollado con el propósito de permitir el control sobre actuadores al operario mediante mando a distancia, entendiendo a Bluetooth como una tecnología inalámbrica, trabajando en una banda de frecuencia sin ninguna restricción.

Mediante este proyecto se evitará el control alambrado y lo más importante es que el operario podrá estar controlando los procesos sin necesidad de estar cerca de la zona donde se está ejecutando el proceso, guardando una distancia segura.

El proyecto además de ejecutar control para un máximo de ocho actuadores, también permite recibir datos de temperatura, donde esta variable sea crítica dentro del proceso y deba supervisar- se constantemente. El sistema tiene dos modos de operación, los cuales son:

- Modo remoto

- Control por computador

Modo remoto se refiere a que el operario puede manejar desde cualquier sitio, siempre y cuando este dentro del rango de trabajo de Bluetooth, el proceso sin necesidad de estar en la estación de control.

Mediante el control por computador el sistema se acopla al PC a través del protocolo RS232 y desde allí se hace el control sobre los procesos.

\section{ANTECEDENTES}

Bluetooth es una tecnología inalámbrica la cual fue desarrollada en la década de los noventa y que actualmente tiene diversos usos, entre los que se destacan el entretenimiento.

Dentro de los trabajos a nivel educativo que se han obtenido con fundamento en esta tecnología se presentan los siguientes:

La investigación titulada: Talking Points, desarrollada recientemente por un grupo de investigadores de la Universidad de Michigan [1], la cual se basa en un sistema de orientación urbana, diseñado primordialmente para personas invidentes, que proporciona una tecnología de información sobre el mundo real, indicándoles los puntos que pueden ser de interés a medida que van recorriendo una ruta específica.

Investigaciones enfocadas hacia las aplicaciones 
de Bluetooth en celulares como Programación de dispositivos Bluetooth a través de Java [2], hacen desarrollos de aplicativos para celulares utilizando plataformas JAVA, de forma que el usuario final pueda reconocer los dispositivos cercanos o servicios que se encuentran dentro del rango de acción del dispositivo Bluetooth.

Por otra parte se han hecho investigaciones sobre el uso de las herramientas MATLAB y Labview como se corrobora en la investigación Implementación del protocolo Bluetooth para la conexión inalámbrica de dispositivos electrónicos programables en la que se realiza una interfaz mediante las herramientas citadas a principio de párrafo, con la finalidad de disponer de tal interfaz a futuro para aplicaciones de robótica instrumentación y control [3].

Donde se ha visto una mayor investigación sobre Bluetooth ha sido en el establecimiento de los enlaces y la forma en que el rendimiento de esta herramienta se ve afectado tanto por interferencias de otras tecnologías como Wi-Fi como también por la distancia de operación. En la investigación Bluetooth voice and data performance in 802.11 DS WLAN environment se trata el rendimiento que tiene una WLAN tanto en los enlaces de voz como en los enlaces de datos, mostrando que los enlaces de voz se ven menos afectados por interferencia cuando se encuentran en un rango de $2 \mathrm{~m}$, mientras que los enlaces de datos pueden tener una reducción del $10 \%$ a una distancia de $10 \mathrm{~m}$ siendo la probabilidad que esto ocurra de un $24 \%$ [4].

Asimismo, la investigación Vehicular Technology también trata de la afectación del rendimiento de los dispositivos Bluetooth por interferencia, pero debido a otras redes Bluetooth presentes dentro del rango de operación de otra red [5].

Se observa que en la mayoría de las investigaciones donde se trabajo algún tipo de prototipo, se debe controlar siempre los dispositivos Bluetooth desde un computador lo cual hace que el radio de acción del dispositivo Bluetooth sea siempre fijo. A diferencia de las investigaciones anteriormente comentadas esta se enfocada hacia el entorno industrial, permite que el radio de acción de los dispositivos Bluetooth sean variables debido al hecho que se ha diseñado una unidad remota que cumple las funciones del computador cuando no se tienen acceso a este componente. Además, permite si se requiere hacer mediciones de la confiabilidad del enlace y se pueden hacer cambios en el modo de operación del dispositivo maestro dependiendo de las necesidades del usuario, permitiendo que el dispositivo pueda tener adaptabilidad a las condiciones que se le presenten. Es por este motivo que el proyecto Sistema electrónico para control de actuadores mediante Bluetooth, tiene en cuenta esta limitante y desarrolla una portabilidad. lo que permite que el radio de acción sea más amplio como se ilustra en la Fig. 1.

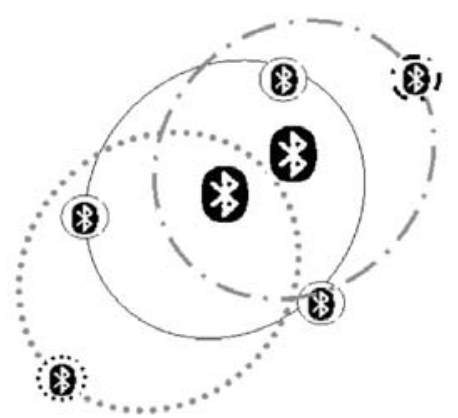

Fig. 1. Radio de acción de un dispositivo portable.

Para lograr este objetivo de portabilidad a fin de que el usuario pudiera tener un radio de acción más amplio, se debía hacer un prototipo el cual pudiera asumir las funciones más importantes desarrolladas por el computador en ausencia de este. Además el dispositivo Bluetooth debía tener un alcance relativamente alto por lo que se recurrió al Parani ESD 110, el cual es un clase 1 y es de fácil configuración [6].

\section{METODOLOGÍA}

En el siguiente apartado se presenta la metodología del diseño del hardware para el sistema de control de actuadores.

\subsection{Diseño del dispositivo}

Definidos los propósitos que debía tener el prototipo se dividió en las siguientes etapas:

- Elaboración de un teclado el cual tiene 2 ca- 
racteres por tecla haciendo uso de la tecla shift, y dos teclas con el único propósito de permitir la conexión o desconexión de las distintas salidas de control (Módulo de entrada).

- Diseño del programa del microcontrolador que permite el manejo del reconocimiento del teclado, la transmisión, recepción y visualización de los datos (Módulo de control).

- Uso de un LCD de 2X16 para permitir visualizar los comandos que se están ejecutando desde el sistema de control hacia las salidas que controlan los actuadores (Módulo de visualización).

- Transmisión de los comandos de control desde el módulo de control hacia la unidad receptora (Módulo de transmisión).

- Recepción de datos enviados por un sensor de temperatura localizado en el módulo esclavo2 y de igual forma cuando se ha hecho la desconexión entre los dispositivos (Módulo de recepción).

La Fig. 2 muestra el diagrama en bloques de la aplicación.

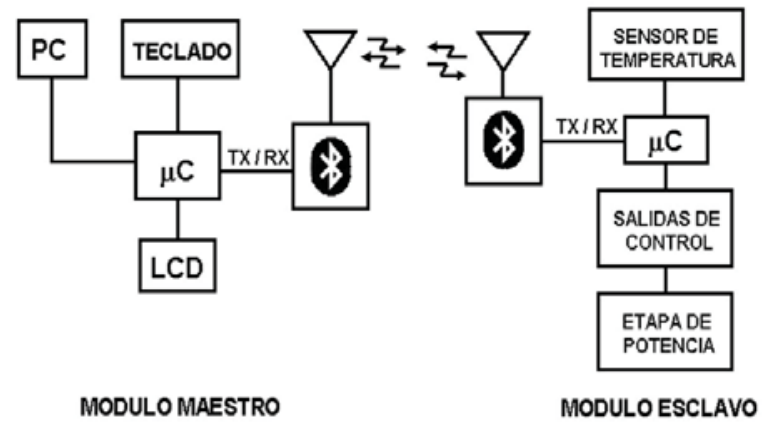

Fig. 2. Diagrama en bloques de la aplicación.

\subsubsection{Módulo de entrada}

Este módulo tiene como fin principal el reconocimiento de los datos ingresados por el usuario a través de un teclado matricial (ver Fig. 3), este se compone de 16 teclas de las cuales 8 tienen doble función, esto con el fin de hacer un uso de un teclado adicional, de tal forma que el sistema final pueda ser lo más compacto y portable posible.

Las teclas de control están designadas por ON y OFF, siendo la tecla Enter la que ejecuta finalmente el proceso de control y conexión del sistema.
Existen también caracteres numéricos los cuales definen la salida o actuador que se requiere controlar.

El teclado por lo tanto tiene caracteres que son solamente necesarios para la conexión y el control sobre los actuadores, de la misma forma que la posterior desconexión de la comunicación.

Es importante resaltar que se debe seguir una serie de comandos denominados AT los cuales reconoce el módulo Bluetooth y que permiten la conexión y desconexión entre dos dispositivos. Existen otros que son de configuración.

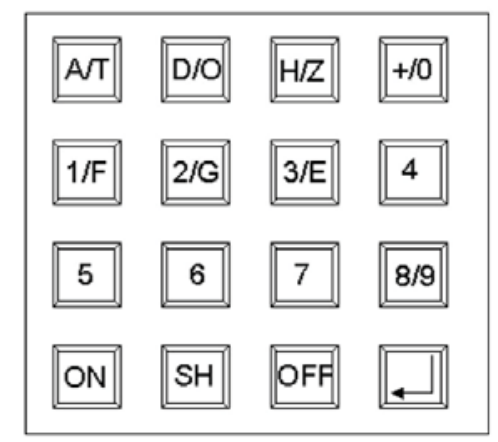

Fig. 3. Teclado de control del sistema.

En pruebas iniciales se diseñó el sistema con dos teclados, sin embargo, se cambió de idea debido al aumento del espacio físico y además que no todas las teclas se utilizaban frecuentemente, por tanto optó por dejar solo un teclado con las funcionalidades que se comentaron anteriormente.

Si en vez de usar el modo remoto, el usuario prefiere el modo de control por computador, el teclado del PC también tendrá destinado dos teclas de control para apagar y prender las salidas que controlan los actuadores y estas serán la tecla punto (.) como ON y la tecla $\left(\_\right.$) como OFF, los números del teclado representarán cada una de las salidas de control, que en esencia es el mismo funcionamiento que con el teclado matricial.

\subsubsection{Módulo de control}

La información ingresada por el usuario a través del teclado habilitado en el sistema, es reconocida por un microcontrolador GP32 el cual ejerce la 
función central del proceso.

Este microcontrolador ejecuta los siguientes procesos en secuencia:

Como primera medida, el microcontrolador reconoce si está habilitado el modo remoto o por el contrario está habilitado el modo de control por computador. En caso de estar habilitado el modo remoto el teclado habilita inmediatamente el reconocimiento del este y la visualización por medio de un LCD.

Vigila constantemente los datos que ingresa el usuario y los muestra por el LCD. En el momento que el usuario presione la tecla Enter, esta información es enviada por medio de la transmisión serial al módulo de Bluetooth (ver Fig. 4) y luego de ello el microcontrolador habilita la recepción serial, esperando una respuesta afirmativa de la aceptación de la instrucción digitada, o, por el contrario, envía una respuesta negativa indicando una instrucción errónea o que simplemente no está listo.

Define el estado del sistema, es decir, si efectivamente ha hecho conexión con el dispositivo que controla los actuadores, esto lo hace mediante un led indicador en el sistema, de tal forma que el usuario pueda saber con exactitud en qué momento el dispositivo ha creado una conexión y de igual modo cuando se ha finalizado la conexión, ya sea por instrucción o por factores externos.

En el Fig. 4 se muestra el diagrama de flujo que detalla el proceso que ejerce el microcontrolador.

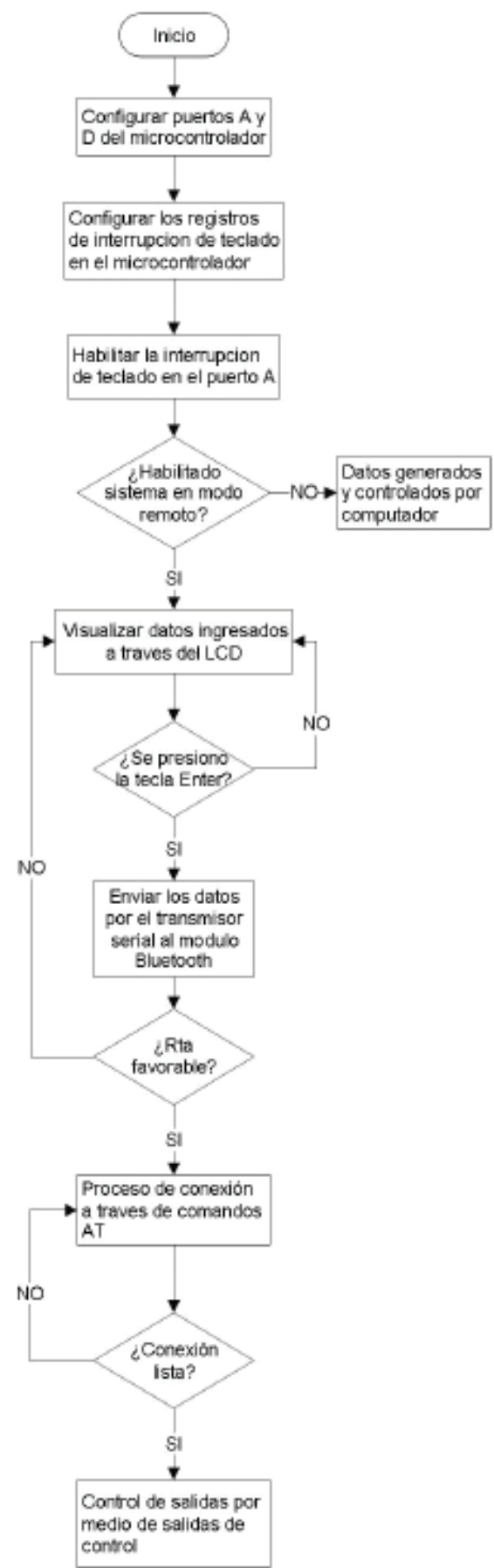

Fig. 4. Diagrama de flujo de funcionamiento de las tareas del microcontrolador para la conexión. 


\subsubsection{Módulo de visualización}

Como existen dos modos de operación, el usuario puede utilizar cuando esté en modo remoto un LCD de 2X16 para visualizar cada uno de los comandos por ejecutar como se visualiza en la Fig. 5.

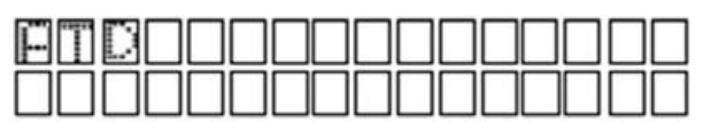

Fig. 5. Visualización de los comandos ejecutados a través de LCD.

O si por el contrario está en modo de control por computador puede ejecutar Hyperterminal y ejecutar los comandos en este, como se muestra en la Fig. 6.

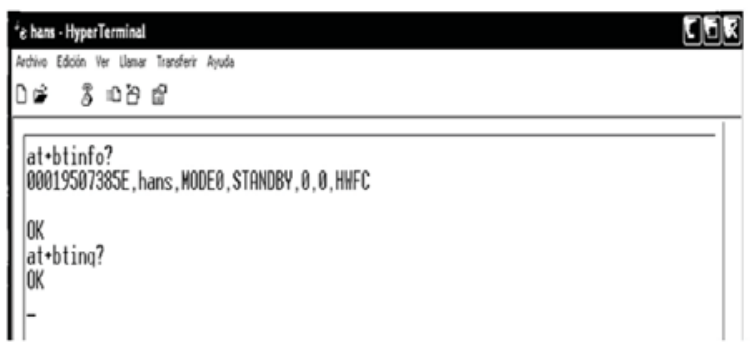

Fig. 6. Visualización de los comandos ejecutados a través del computador.

\subsubsection{Módulo de transmisión y ción}

recep-

Este módulo es uno de los más importantes por cuanto permite la comunicación correcta con el módulo Bluetooth y por consiguiente con cualquier dispositivo con el que se quiera realizar la conexión.

Es de vital importancia configurar los parámetros necesarios para que tanto el microcontrolador como el módulo de Bluetooth se puedan comunicar. Dentro de estos parámetros se encuentran los siguientes:
- Velocidad de transmisión

- Paridad

- Bit de parada

- Control de flujo

Con el microcontrolador GP32 se configuran estos parámetros mediante registros dedicados exclusivamente para las comunicaciones seriales. Por otra parte con el uso de comandos AT se configura el módulo de Bluetooth con el siguiente comando:

$$
\begin{aligned}
& \text { AT+UARTCONFIG, } b, p, s, h \\
& \text { Donde: } \\
& \mathrm{b}=\text { Baud rate, usualmente } 9600 \\
& \mathrm{p}=\text { Paridad } \rightarrow N=\text { none } \\
& \rightarrow E=\text { Even } \\
& \rightarrow O=\text { Odd } \\
& \mathrm{s}=\text { bit de parada } \rightarrow 1 \text { bit de parada } \\
& \rightarrow 2 \text { bits de parada } \\
& \mathrm{h}=\text { Control de flujo } \rightarrow \mathrm{NO} \\
& \rightarrow S I
\end{aligned}
$$

Debe existir entre los dos dispositivos los mismos parámetros de configuración de la comunicación serial para establecer correctamente la comunicación de tal forma que los datos lleguen a salvo.

En esencia el proceso de comunicación serial para el microcontrolador y el módulo Bluetooth es enviar una información y esperar la confirmación de esa información, por tanto el microcontrolador está cambiando su función de transmisor a receptor alternativamente, en función de la repuesta generada por el módulo Bluetooth.

\section{MÓDULO DE POTENCIA}

Este se encuentra en el módulo esclavo (Fig. 2).

Está compuesto inicialmente de una etapa de opto acople con el fin de aislar eléctricamente la etapa donde se encuentra el dispositivo Bluetooth. La salida de potencia es salida a transistor como se muestra en la Fig. 7. 


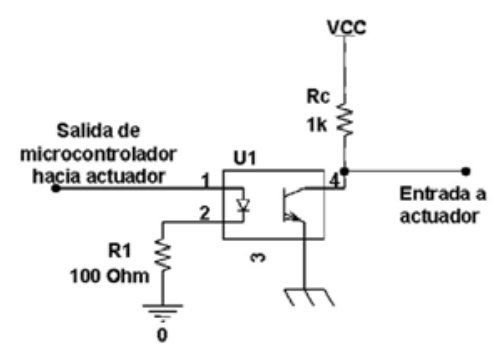

Fig. 7. Etapa de potencia para una actuador.

\section{RESULTADOS}

$\mathrm{Al}$ estar configuradas tanto la tarjeta transmisora manejada por el usuario como la tarjeta receptora manejada por los actuadores, (que además opera la variable temperatura), se procedió a establecer la comunicación.

Las pruebas de comunicación se hicieron con dos módulos Bluetooth ESD 110 [6] los cuales son clase 1 y su distancia de trabajo nominal según el fabricante es de $100 \mathrm{~m}$.

Las pruebas se realizaron en un entorno cerrado, en laboratorios de electrónica bajo un ambiente controlado y en donde los dispositivos maestros y esclavo estaban separados por tres laboratorios en medio de su trayectoria de enlace.

Haciendo pruebas entonces para entorno abierto y cerrado se obtuvieron los resultados descritos en las Tablas 1 y 2 .

Tabla 1. Mediciones realizadas en entornos cerrados.

\begin{tabular}{|c|c|}
\hline Distancia $(\mathrm{m})$ & Conexión \\
\hline 4 & Exitosa \\
\hline 10 & Exitosa \\
\hline 20 & Exitosa \\
\hline 30 & Exitosa \\
\hline 40 & Exitosa \\
\hline 45 & Exitosa \\
\hline 50 & Exitosa \\
\hline 55 & Exitosa \\
\hline 60 & Exitosa \\
\hline 70 & Relativa \\
\hline 80 & Fallida \\
\hline
\end{tabular}

Nota: Conexión relativa se refiere a la aleatoriedad de las conexiones exitosas o fallidas que tuvo la comunicación a determinada distancia.

Tabla 2. Tiempos de conexión según distancia.

\begin{tabular}{|c|c|}
\hline Distancia $(\mathrm{m})$ & Tiempo de conexión $(\mathrm{seg})$ \\
\hline 10 & 2 \\
\hline 20 & 2 \\
\hline 30 & 3 \\
\hline 50 & 5 \\
\hline 70 & 8 \\
\hline
\end{tabular}

Tabla 3. Mediciones realizadas con dispositivos dispuestos en línea de vista bajo incidencia de fuentes de ruido eléctrico.

\begin{tabular}{|c|c|}
\hline Distancia $(\mathrm{m})$ & Conexión \\
\hline 6 & Exitosa \\
\hline 12 & Exitosa \\
\hline 18 & Exitosa \\
\hline 24 & Exitosa \\
\hline 30 & Exitosa \\
\hline 36 & Exitosa \\
\hline 42 & Exitosa \\
\hline 48 & Exitosa \\
\hline 54 & Exitosa \\
\hline 60 & Exitosa \\
\hline 66 & Exitosa \\
\hline 72 & Exitosa \\
\hline 78 & Exitosa \\
\hline 84 & Relativa \\
\hline 90 & Relativa \\
\hline 93 & Fallida \\
\hline 100 & Fallida \\
\hline
\end{tabular}

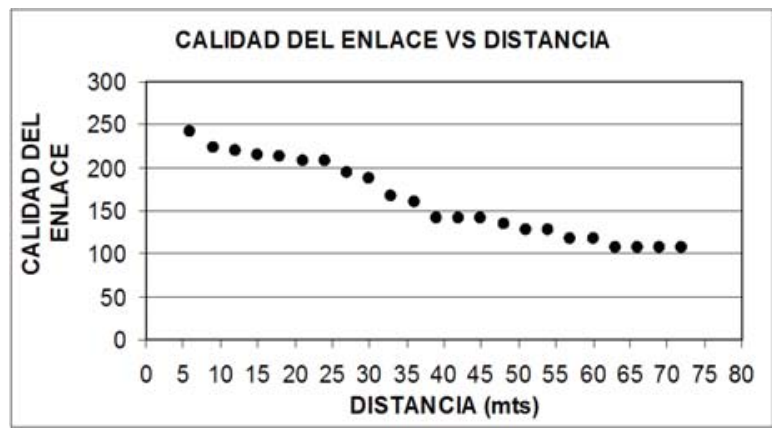

Fig. 8. Gráfico de calidad de fuerza del enlace vs distancia bajo condiciones de entornos con ruido eléctrico.

Nota: El valor óptimo de calidad de enlace está 


\section{re-creaciones}

valorado en 255 y cualquier disminución del valor en esta gráfica representa un debilitamiento en la fuerza de la señal y por tanto en su calidad.

A medida que se fue aumentando la distancia entre el Bluetooth en modo maestro y el Bluetooth en modo esclavo, se tuvo mayor dificultades en la conexión por cuanto se presenta un rango mayor de dispositivos en el área de enlace de los dos dispositivos. Cabe anotar que los dispositivos Bluetooth trabajan en la Banda ISM la cual es ampliamente utilizada y por lo que se hace más difícil establecer una conexión segura haciendo que esto demore el proceso de enlace de los dos dispositivos, mientras que cuando los dispositivos se encontraban a una distancia menor a $10 \mathrm{~m}$ la conexión era exitosa y el tiempo de establecer la conexión era corto, como se puede observar en los resultado de las Tablas 1 y 3.

\section{CONCLUSIONES}

El sistema en modo remoto permite el desarrollo de las funciones de conexión, desconexión y de control sobre los actuadores, sin embargo, desde este modo no se pueden hacer tareas de configuración del dispositivo como por ejemplo cambiar de modo maestro a modo esclavo, o establecer los parámetros de la comunicación serial, debido a la evaluación de espacio físico vs dispositivo compacto que fuera portable. Cuando se maneja el dispositivo por computador se pueden hacer configuraciones al dispositivo.

La distancia nominal que se plantea en la hoja de datos del dispositivo Bluetooth, debe revaluarse tanto para comunicaciones en entornos abiertos como para entornos cerrados, ya que si los hubiese el dispositivo no alcanza este radio de acción como se pudo observar en las tablas de mediciones 1 y 3 .

El dispositivo hecho puede ser una herramienta para el control de diferentes actuadores, sin que ello signifique que siempre el dispositivo tenga que realizar esta función. Más bien este dispositivo es una ayuda redundante cuando el operario no se encuentre en la estación de control.

\section{TRABAJOS FUTUROS}

Se plantea a futuro permitir que desde el módulo maestro se puedan enviar comandos al módulo esclavo los cuales le permitan que en posteriores conexiones este últimó se pueda comportar como maestro con el fin de que cuando se presente alguna alerta este módulo se comunique con el módulo central advirtiendo sobre una posible falla en algún sistema.

\section{FINANCIAMIENTO}

El proyecto presentado a través de este artículo se enfocó a observar la aplicabilidad del uso de redes Bluetooth en los ambientes industriales, basado en investigaciones previas que se enfocaban hacia los métodos de comunicación y aplicaciones haciendo uso de esta herramienta. Sin embargo, este proyecto fue financiado con recursos propios.

\section{REFERENCIAS}

[1] Michigan University. Proyecto talking points. [En línea], Available: http://www. eurekalert.org/pub_releases/2008-09/ uom-nbs091808.php

[2] J. C. Haartsen, S. Zürbes, "Bluetooth voice and data performance in 802.11 DS WLAN environment", SIG Publication, 1999. [En línea], Disponible en: http:// infotooth.tripod.com/documents/Bluetooth_voice_and_data_performance_
in_802.11DS_WLAN_environment.pdf

[3] Java hispano. JSR-82: Bluetooth desde Java. [En línea], Disponible en: http:// www.javahispano.org/contenidos/es/ jsr82_bluetooth_desde_java/

[4] R. Linares, J. Vásquez, G. Holguín. “Implementación del protocolo Bluetooth para la conexión inalámbrica de dispositivos electrónicos programables", Scientia et Técnica, no. 24, pp. 31-36, 2004. 
re-creaciones

[5] I. Howitt, "Mutual Interference Between Independent Bluetooth Piconets", IEEE Transaction on Vehicular Technology, vol. 52, no.3, pp. 708-718, May. 2003.
[6] Manual de usuario, ParaniESD100/110/200/210 User Guide Version 1.0.3. Sena Tecnologies. 2006. 